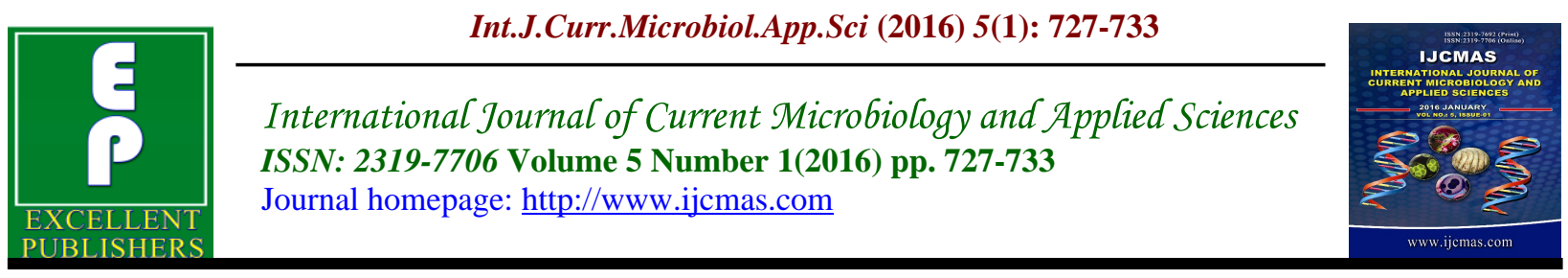

Original Research Article

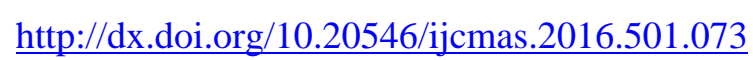

\title{
Outbreak of Carbapenemase-Producing Klebsiella pneumoniae Blood Stream Infections in Neonatal Intensive Care Unit
}

\author{
Jaspal Kaur*, Sheevani Sheemar, Kailash Chand, Shashi Chopra And Gomty Mahajan \\ Department of Microbiology, Punjab Institute of Medical Sciences, Jalandhar, India
}

*Corresponding author

\begin{abstract}
A B S T R A C T
Keywords

Blood stream infections,

Klebsiella pneumoniae, neonatal intensive care unit

\section{Article Info}

Accepted: 25 December 2015 Available Online: 10 January 2016

Neonatal sepsis forms the most common cause of mortality in the first month of life in developing countries. Klebsiella species are the most commonly implicated pathogen in neonatal sepsis. Carbapenem-resistant Klebsiella pneumoniae is associated with high morbidity and mortality. The study was undertaken in the department of Microbiology, Punjab Institute of Medical Sciences, Jalandhar when in the month of August 2014, a sudden rise in positive blood cultures due to phenotypically identical Klebsiella pneumoniae from samples received from neonatal intensive care unit raised an alarm. Out of total 23 blood culture samples obtained from neonates, 12 were positive for Klebsiella pneumoniae subspecies aerogenes. Outbreak investigation revealed the similar growth from 3 different sites of NICU (2 from work surfaces and 1 from medicine trolley). Antibiogram typing revealed that all the isolates belonged to a single strain. The strain was multidrug resistant and tested positive for carbapenemase production by Modified Hodge test. The main cause behind outbreak was found to be breach in infection control measures. The study focuses on the prevention of nosocomial outbreaks by stringent infection control measures and judicious use of antibiotics in hospital settings.
\end{abstract}

\section{Introduction}

Klebsiella pneumoniae is a well recognized cause of community and hospital acquired neonatal infections in developing countries with case fatality rate varying from $18 \%$ to $68 \%$ (Saleem et al., 2013). Infants, particularly those with a low gestational age or a low birth weight, are at greater risk for developing nosocomial infections in neonatal intensive care units (NICUs) due to their prolonged hospital stays, exposure to central venous catheters, mechanical ventilation, total parenteral nutrition, and prolonged administration of antibiotics. Mutidrug-resistant Klebsiella pneumoniae sepsis has serious implications because of the limited choices of antibiotics available, increased hospital stay and expenditure, and poor neonatal outcome (Mumbula et al., 
2015). Carbapenem-resistant Klebsiella pneumoniae is associated with high morbidity and mortality (Roy et al, 2011, Jin et al., 2015). The Klebsiella species, in addition to its virulence and ability to acquire antibiotic resistance determinants are able to survive on skin and watery surfaces and resist desiccation which adds to its pathogenicity. The contaminated environment serve as a reservoir for this pathogen, making it easily transferable through equipments and the hands of health care workers. So, one of the biggest factor contributing in nosocomial outbreaks is poor adherence to infection controlpolicies in health-care settings. The study here is undertaken to report a nosocomial outbreak of multidrug resistant carbapenemaseproducing Klebsiella pneumoniae blood stream infections in neonatal intensive care unit(NICU) of our hospital and measures taken to curtail it.

\section{Materials and Methods}

The study was undertaken in the department of Microbiology, Punjab Institute of Medical Sciences, Jalandhar when in the month of August2014, a sudden rise in positive blood cultures from samples received from neonatal intensive care unit due to phenotypically identical Klebsiella pneumoniae raised an alarm. By this time 6 phenotypically identical Klebsiella pneumoniae species had already been isolated from 13 blood culture samples received from NICU.

The study was further stretched over a period of one month to know all the cases which suffered from the outbreak. A probable case was defined as an infant aged $<28$ dayswho was admitted to the NICU between August 2014 and September 2014 and presenting with clinical signs of blood stream infection and having blood culture positive for Klebsiella species. Clinical signs of infection which were studied included poor feeding, lethargy, respiratory problems likes tachypnea, apnea, grunting and cyanosis, hyperthermia, gastrointestinal problems like vomiting, abdominal distension, diarrhoea, and central nervous system symptoms including convulsions, hypotonia, and irritability.

Samples for blood culture were received in brain heart infusion broth. Repeated subcultures were done on 5\% sheep Blood agar and Mac-Conkey agar after 24 hours, 48 hours, 72 hours and 7 days of incubation at $37^{\circ} \mathrm{C}$. Isolates from positive cultures were identified by standard biochemical procedures (Collee et al., 2006). Cultures showing growth of Klebsiella pneumoniae were included in the study.

\section{Antimicrobial Susceptibility}

The antibiotic susceptibility testing was done by Kirby-Bauer disc diffusion test according to CLSI (Clinical and Laboratory Standards Institute, 2015) guidelines. The strain was typed on the basis of Antibiogram. Unfortunately, molecular typing could not be done due to lack of facilities. Antibiotics used were cefotaxime $(30 \mu \mathrm{g})$, cefpodoxime $(30 \mu \mathrm{g})$,ceftrioxone $(30 \mu \mathrm{g})$, cephoxitin $(30 \mu \mathrm{g})$, chloramphenicol $(30 \mu \mathrm{g})$, cotrimoxazole $(30 \mu \mathrm{g})$, gentamicin $(10 \mu \mathrm{g})$, amikacin $(30 \mu \mathrm{g})$, ciprofloxacin $(5 \mu \mathrm{g})$, amoxicillin/clavulanate $(20 / 10 \mu \mathrm{g})$ piperacillin/tazobactam $(100 / 10 \quad \mu \mathrm{g})$, meropenem $(10 \mu \mathrm{g}), \quad$ imipenem $(10 \mu \mathrm{g})$,

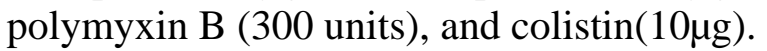

All the antibiotic discs were procured from Hi-media, Mumbai.The strains were tested for ESBL production by Phenotypic confirmatory disc diffusion test (PCDDT), AmpC production by AmpC disk test and Carbapenemase production by Modified Hodge test. 


\section{Phenotypic Confirmatory Disc Diffusion Test (PCDDT)}

This test was performed as per CLSI guidelines by using control strains of Escherichia coli ATCC 25922 (betalactamase negative) and Klebsiella pneumoniae ATCC 700603 (ESBL positive).A $>=5 \mathrm{~mm}$ increase in zone diameter for either ceftazidime or cefotaxime tested in combination with clavulanic acid versus its zone diameter when tested alone confirms ESBL production.

AmpC Disk Test (Black et al., 2005)

A lawn culture of a 0.5 McFarland's suspension of ATCC Escherichia coli25922 was prepared on a Mueller-Hinton agar plate. A $30 \mu \mathrm{g}$ cephoxitin disc was placed on the inoculated surface of the agar. A sterile plain disc $(6 \mathrm{~mm})$ which was inoculated with several colonies of the test organism was placed beside the cephoxitin disc, almost touching it. After an overnight incubation at $37^{\circ} \mathrm{C}$, the plates were examined for either an indentation or a flattening of the zone of inhibition, which indicates the enzyme inactivation of cephoxitin (positive result), or an absence of distortion, which indicates no significant inactivation of cephoxitin (negative result).

Modified Hodge Test (Ramana et al., 2013)

The strains were subjected to Modified Hodge test for detection of carbapenemases. An overnight culture suspension of Escherichia coli ATCC 25922 adjusted to $0.5 \mathrm{McF}$ arland standard was inoculated using a sterile cotton swab on the surface of a Mueller-Hinton agar. After drying, $10 \mu \mathrm{g}$ meropenem disk was placed at the center of the plate and the test strains were streaked from the edge of the disk to the periphery of the plate in four different directions. The plate was incubated overnight at $37^{\circ} \mathrm{C}$. The presence of a 'cloverleaf shaped' zone of inhibition due to carbapenemase production by the test strains was considered as positive.

\section{Outbreak Investigation}

To trace out the possible source of this outbreak, a team headed by a microbiologist (member, infection control committee) visited the unit and specimens were collected from different possible sources of infection including environmental samples, samples from instruments and hands of nursing staff. All the samples were collected in trypticase soy broth (TSB) and subcultured after overnight incubation on MacConkey agar and Blood agar and checked for the growth of Klebsiella pneumoniae having same biochemical profile and sensitivity pattern as was seen in the cases. Staff of NICU was interviewed to ascertain the compliance to the infection control policies and to evaluate any change in routine procedures that could have contributed to the outbreak. The staff was instructed to adopt strict antiseptic techniques. Proper sterilization \& disinfection of instruments and NICU was advised. NICU was temporarily shut down for fumigation.

\section{Follow Up}

Follow up of all the blood culture samples received from NICU was done for an extended period of six months i.e. till March 2015 as a precautionary measure and to ascertain the execution of infective control measures.

\section{Results and Discussion}

Out of total 23 blood culture samples 
obtained from neonates, 12 were positive for Klebsiella pneumoniae subspecies aerogenes. Apart from this 3 environmental samples taken from different sites of NICU(2 from work surfaces and 1 from medicine trolley) revealed the similar growth (Table 1).Antibiogram typing revealed that all the isolates belonged to a single strain. The srain was resistant to all the cephalosporins, aminoglycosides, fluoquinolones, $\quad \beta$-lactam+ $\beta$-lactamase inhibitor combination drugs and even to carbapenems. The only drug which was sensitive in all the isolates was colistin. The isolates tested negative for ESBL and AmpC production by PCCDT and AmpC disk tests respectively. However, Modified Hodge test for carbapenemase production was positive with 'cloverleaf shaped' zone of inhibition around meropenem disc.

Prematurity and low birth weight was the most frequent underlying condition of the infected neonates. Out of 12 neonates, whose blood cultures was positive for Klebsiella pneumoniae, 8 were low birth weight, with birth weights ranging from $1200 \mathrm{~g}$ to $2,300 \mathrm{~g}$. Six neonates were preterm with gestational age ranging from 26weeks to36 weeks. Male to female ratio was $2: 1$. All the neonates had a history of empiric antibiotic therapy with a third generation cephalosporin (cefotaxime) and an aminoglycoside (amikacin). The main symptoms were respiratory problem (tachypnea), poor feeding and lethargy with two neonates having associated diarrhoea and four having jaundice. C-reactive protein levels were raised in all the cases. After culture and sensitivity report, the empiric treatment was changed to colistin. However, two neonates succumbed to infection. Anxious relatives of two patients shifted their kids to some other hospital against medical advise and so they could not be followed up. Rest of 8 neonates responded to treatment and survived and later on discharged.

Sepsis, in the first month of life still forms a major cause of mortality and morbidity in the developing countries like India. Multidrug resistant gram-negative organisms form a big threat to the preterm, low birth weight and immuocompromised newborns. Klebsiella species are the most commonly implicated pathogen in neonatal sepsis outbreaks followed by Escherichia coli(Szilagyi et al., 2010, Roy et al., 2011). However, Enterobacter species outbreaks have also been reported (Ostwal et al.,2014).

Neonatal septicaemia is difficult to diagnose clinically as it presents with non-specific signs and symptoms. The main symptoms in our study were respiratory problem (tachypnea), poor feeding and lethargy. Two neonates had associated diarrhoea and four had jaundice.

There can be intrinsic risk factors for infection of newborns like gestational age, sex, birth weight and immunologic development (Zakariya, 2015). In our study, prematurity and low birth weight was the main underlying condition of the infected neonates. Prematurity is the leading cause of neonatal sepsis in other studies also (Rastogi et al. 2010, Fabbri et al., 2013). Immature immune system in preterm neonates predisposes them to infection. Majority of cases were males. Higher rate of sepsis in male newborns has been reported in other studies also (Saleem et al., 2013). This can be due to gender biasing regarding care-seeking in illness in newborns in countries like India (Willis JR et al., 2009).

The extrinsic factors which generally contribute to outbreak are prolonged hospital stay, use of invasive procedures, exposure to hospital environment and staff, 
overcrowding, understaffing, breach in hygiene and hospital infection control techniques and injudicious use of antimicrobials. In our instituition, understaffing and inadequate practising of infection control measures probably led to the outbreak. The contaminated environment can serve as reservoirs for this pathogen, which is then spread by health care worker's hand carriage (Gupta et al., 2004). In an earlier report of outbreak of sepicaemia in neonatal ICU in India due to multidrug resistant K.pneumoniae, the phenotypically identical K.pneumoniae strains were isolated from disinfectant solution, suction fluid, water tap and hands of HCWs (Rastogi et al. 2010,).While investigating the outbreak, we isolated the implicated pathogen from work surfaces and medicine trolley. In NICU, there is compulsion for hand washing \& wearing gowns while entering into unit, but we found there was breach in infection control measures. The main culprits found were the trainee nursing students and General duty assistant (GDA) posted in the unit who were not well versed with the infection control measures to be taken in an intensive care unit. On inquiring the nurse in-charge, it was found that due to shortage of GDA staff, a single GDA posted in NICU was also shared with some other ward and hence he frequently moved in and out of the unit without wearing gowns and taking other necessary precautions. Nursing students were found negligent regarding hand washing protocol. The staff was instructed to adopt strict antiseptic techniques with particular emphasis on compliance with hand disinfection protocol between all contacts with patients. Proper sterilization \& disinfection of instruments and NICU was advised.

Table.1 Organisms Isolated from Samples Taken from Different Sites of NICU

\begin{tabular}{|l|l|}
\hline Sample site( no of samples) & Organisms isolated \\
\hline Phototherapy unit (2) & No growth \\
\hline Incubators (2) & No growth \\
\hline Medicine trolley (2) & Klebsiellapneumonia \\
\hline Work surfaces (3) & Klebsiellapneumoniae(2) \\
\hline Sink (1) & Aerobic spore bearer \\
\hline Suction apparatus(2) & Aerobic spore bearer \\
\hline Hands of nursing staff (5) & $\begin{array}{l}\text { Coagulase negative } \\
\text { micrococci(2) }\end{array}$ \\
\hline Weighing scale(1) & Aerobic spore bearer \\
\hline Room air & Fungus+ Coagulase negative Staphylococcus \\
\hline
\end{tabular}

An important compounding factor in the treatment of these infections is the emergence of multidrug resistance which limits the treatment options (Krishna et al., 2007, Ali et al., 2012, Fabbri et al., 2013). To add to the grievances of debilitated neonates, Klebsiella pneumoniae strain implicated in the outbreak turned out to be multidrug resistant and resistant even to the last treatment options i.e. carbapenems. Modified Hodge test showed the strain to be carbapenemase producing. Carbapenemases hydrolyse most $\beta$-lactams. They are associated with diverse mobile genetic elements carrying multiple resistancegenes, thereby conferring resistance to several 
antibioticclasses, such as aminoglycosides, fluoroquinolones, tetracyclines, trimethoprim, and sulphonamides .The only drug which was found sensitive in our case was colistin and hence the only treatment option available.

In conclusion, Klebsiella pneumoniae has been reported as one of most frequent causes of infection outbreaks in neonatal intensive care units. Neonatal infections due to multidrug resistant gram negative organisms are of grave concern due limitations in treatment options. Carbapenems are the last resort for the treatment of such infections, however, the emergence of carbapenemases has further narrowed down the treatment options to polymyxins. So, the main focus should be on the prevention of these deadly infections. This study is reported with the main aim of highlighting the importance of infection control measures. A slight breach in infection control measures can wreak havoc especially in NICUs where weak, fragile, premature and low birth weight babies easily fall prey to infection by virulent multidrug resistant organisms. Further, prompt action should be taken once such outbreaks are suspected for early detection and better treatment outcomes. To eliminate such outbreaks, stringent infection control measures such as proper handwashing practices, improved aseptic technique for clinical procedures and a general awareness of infection control and antibioticpolicies should be instituted. Judicious use of carbapenems can prevent the rise in cabapenem resistant isolates.

\section{Acknowledgement}

Sincere thanks to Dr.Jatinder Singh, Associate Professor, Department of Pediatrics for providing relevant information about patients and helping in investigating and controlling the outbreak.

\section{References}

Ali S, Abbasi SA, Mirza IA, Khan IU, Fayyaz M, Hussain A, Satti L. 2012 Outbreak of multidrug-resistant Klebsiella pneumoniae sepsis in Neonatal Intensive Care Unit. Gomal Journal of Medical Sciences., Vol. 10, No. 1

Black JA, Moland ES, Thomson KS. 2005. AmpC Disk Test for detection of plasmid-mediated AmpC $\beta$-lactamase in Enterobacteriacae lacking chromosomal AmpC $\beta$-lactamase. $J$ Clin Microbiol., 43 (7):3110-13.

Collee, JG, Fraser AG, Marmion BP, Simmons, MackeyA. 2006. McCartney practical medical microbiology. In: Collee, J.G., Miles, R.S., Watt, B., (Eds). Tests for the identification of Bacteria, 14th edn. Elsevier, New Delhi, India. Pp. 131-49.

Clinical and Laboratory Standards Institute. 2015.Performance standards for antimicrobial susceptibility testing; 25 th informational supplement M100-S25.

Fabbri G, Panico M, Dallolio L, Suzzi R, MatildeCiccia M, Sandri F et al. 2013. Outbreak of Ampicillin/PiperacillinResistant Klebsiella pneumoniae in a Neonatal Intensive Care Unit (NICU): Investigation and Control Measures Int. J. Environ. Res. Public Health.,10: 808815

Gupta A, Latta PD, Todd B, Gabriel PS , Haas J, Wu F, et al . 2004. Outbreak of Extended Spectrum beta-lactamase producing Klebsiella pneumoniae in Neonatal Intensive Care Unitlinked to artificial nails. Infect Control Hosp Epidemiol., 25:210-215.

Jin Y, Shao C, Li J, Fan H, Bai Y, Wang Y.2015 Outbreak of Multidrug Resistant NDM-1-Producing Klebsiella pneumoniae from a Neonatal Unit in 
Shandong Province, China. Plos one., 10(3): e0119571

Krishna BVS, Patil AB, Chandrasekhar MR. 2007. Extended Spectrum betaLactamase Producing Klebsiella pneumoniae in Neonatal Intensive Care Unit. Indian J Pediatr., 74 (7): 627-630.

Mumbula EM, Kwenda G, Samutela MT, et.al, 2015. Extended Spectrum $\beta$ Lactamases Producing Klebsiella pneumoniae from the Neonatal Intensive Care Unit at the University Teaching Hospital in Lusaka, Zambia. Jour of Med Sc\&Tec., 4(2); 85 - 91.

Ostwal KI, Joshi TA, Kadam PA, Karkhele RR, Iravane JA, Deshmukh LS, et al. 2014Outbreak of Neonatal Septicemia at a Tertiary care Hospital due toEnterobacter cloacae J Dent Med Sci., 13(11):9-12.

Ramana KV, Rao R, Sharada C, Kareem MA, Reddy LR, Ratna Mani MS. 2013. Modified Hodge test: A useful and the low cost phenotypic method for detection of carbapenemase producers in Enterobacteriaceae members. $J$ Nat ScBiol Med., 4: 346-8.

Rastogi V, Nirwan PS, Jain S, Kapil A. 2010. Nosocomial outbreak of septicaemia in neonatal intensive care unit due to extended spectrum $\beta$ lactamase producing Klebsiella pneumoniae showing multiple mechanisms of drug resistance. Indian $J$ Med Microbiol., 28: 380-4.

Roy S, Gaind R, Chellani H, Mohanty S, Datta S, Singh AK, Basu S. 2013.
Neonatal septicaemia caused by diverse clones of Klebsiella pneumoniae \& Escherichia coli harbouringbla ${ }_{\text {СТХ-M-15 }}$ Indian J Med Res.,137: 791-799.

Roy S, Viswanathan R, Arun K. Singh AK, Das P, Basu S. 2011. Sepsis in neonates due to imipenem resistant Klebsiella pneumoniae producing NDM-1 in India. J Antimicrob Chemother., 14111413

Saleem AF, Qamar FN, Shahzad H, Qadir M, Zaidi AKM. 2013.Trends in antibiotic susceptibility and incidence of late-onset Klebsiella pneumoniae neonatal sepsis over a six-year period in a neonatal intensive care unit in Karachi, Pakistan.Int J Infect Dis.,17 :e961-e965.

Szilagyi E, Fuji M, Damjanova V,Boroczi K,Szonyi K, Toth A, Nagy K. 2010 Investigation of Extended Spectrum beta-Lactamase Producing Klebsiella pneumoniae in Hungary between 2005 and 2008.ActaMicrobiologica et ImmunologicaHungarica.,5, 43-53

Zakariya BP, Bhat BV, Harish B N, Babu T A, Joseph NM. 2012. Risk factors and outcome of Klebsiella pneumonia sepsis among Newborns Curr Pediatr Res.,16 (2): $115-118$.

Willis JR, Kumar V, Mohanty S, Singh P, Singh V, Baqui AH, et al. 2009. Gender differences in perception and careseeking for illness of newborns in rural Uttar Pradesh, India. $J$ Health PopulNutr;27: 62-71.

\section{How to cite this article:}

Jaspal Kaur, Sheevani Sheemar, Kailash Chand, Shashi Chopra and Gomty Mahajan.2016. Outbreak of Carbapenemase-Producing Klebsiella pneumoniae Blood Stream Infections in Neonatal Intensive Care Unit. Int.J.Curr.Microbiol.App.Sci. 5(1): 727-733 hittp:///dx.doi.org/10.20546/ijcmas.2016.501.073 